\title{
Autonomous River Exploration
}

Sezal Jain, Stephen Nuske, Andrew Chambers, Luke Yoder, Hugh Cover, Lyle Chamberlain, Sebastian Scherer and Sanjiv Singh

\begin{abstract}
Mapping a rivers course and width provides valuable information to help understand the ecology, topology and health of a particular environment. Such maps can also be useful to determine whether specific surface vessels can traverse the rivers. While rivers can be mapped from satellite imagery, the presence of vegetation, sometimes so thick that the canopy completely occludes the river, complicates the process of mapping. Here we propose the use of a micro air vehicle flying under the canopy to create accurate maps of the environment. We study and present a system that can autonomously explore rivers without any prior information, and demonstrate an algorithm that can guide the vehicle based upon local sensors mounted on board the flying vehicle that can perceive the river, bank and obstacles. Our field experiments demonstrate what we believe is the first autonomous exploration of rivers by an autonomous vehicle. We show the 3D maps produced by our system over runs of 100-450 meters in length and compare guidance decisions made by our system to those made by a human piloting a boat carrying our system over multiple kilometers.
\end{abstract}

\section{Introduction}

Riverine systems are an increasingly important focus for many applications like mapping, monitoring and surveillance where it is desirable to use autonomous exploration to traverse the river and collect up to date information. A small lightweight system that can travel below the tree line to sense the river width, the river direction and canopy clearance is advantageous since this information is often not possible to measure from satellite imagery because tree canopy cover occludes the river from

Robotics Institute, Carnegie Mellon University, 5000 Forbes Ave, Pittsburgh, PA, 15213, USA. sezal@andrew.cmu.edu,nuske@cmu.edu 
above. Further, narrow, densely-forested, rivers are difficult to navigate by surface craft because of submerged and semi-submerged obstacles and therefore we develop a Micro Aerial Vehicle (MAV) that is small and nimble and able to traverse the difficult terrain.

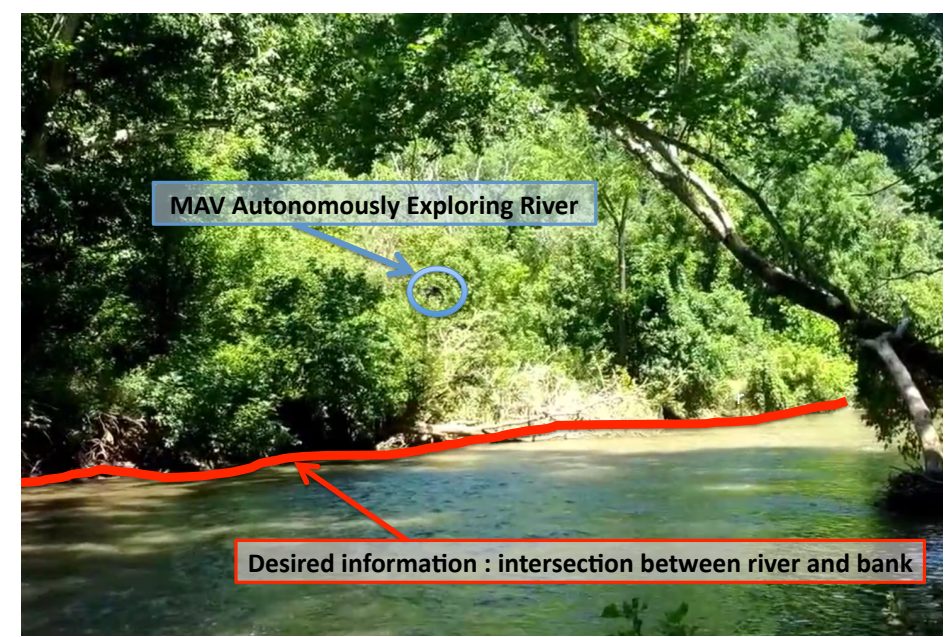

(a) Micro Aerial Vehicle (MAV) autonomously navigating river environment.

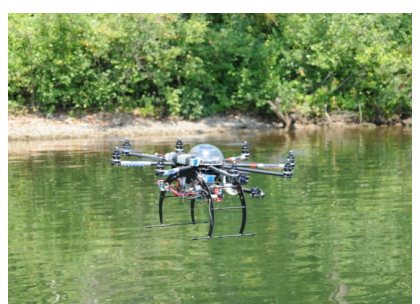

(b) Close-up of vehicle exploring river environment.

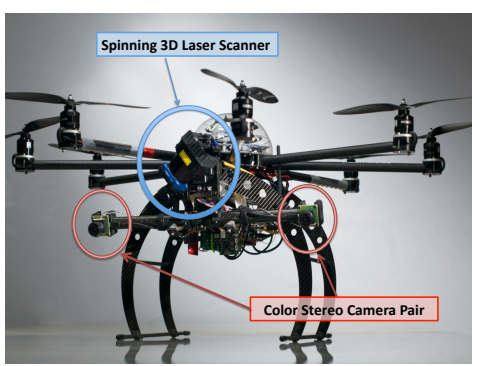

(c) Vehicle local sensing consists of a lightweight spinning laser scanner and stereo color camera pair.

Fig. 1 A Micro Aerial Vehicle is used to autonomously explore and map the river environment. The information of interest is the intersection between bank and river. The vehicle is lightweight and agile and not suseptible to submerged and semi-submerged obstacles such as would be hazardous to a surface vehicle. To avoid obstacles and to perceive the extent and course of the river the vehicle is fitted with a spinning 3D laser scanner and color stereo pair.

Existing applications for autonomous river operations focus on collecting information from robotic boats navigating based on stored directions. These existing 
works have used pre-determined GPS waypoints as a navigation guide. Often riverine systems are densely overgrown with vegetation and autonomous exploration cannot depend on the irregular and erroneous GPS measurements in these surroundings. Due to their dense canopy, estimating an initial map of the waterways from satellite images is also not a viable option. Further riverine environments are also continuously evolving, and current information of the width and course is often not available. For all of these reasons river environments must be explored without relying on pre-determined maps or waypoints. To date, there has not been a truly autonomous exploration system demonstrated in a river environment. We present what we believe is the first such system that can explore a river solely from local sensing, and in addition our vehicle is the first that can fly through river environments to quickly explore while avoiding submerged and semi-submerged obstacles.

We build on our existing work in river environments for autonomous perception, positioning, and obstacle avoidance work $[1,3,4,12,11]$ and extend to add the key capability of truly autonomous exploration. Our contribution here is to present a new exploration algorithm based on a multi-variate cost function to maximize the information collected in a river map given the fixed duration of a mission. We use two sensor modailities which are different in range and accuracy, namely vision and laser in local sensing for the proposed algorithm. We demonstrate that our method is more adept than traditional exploration algorithms and can traverse the river to gather more information during a mission.

\section{Related Work}

Much work has been placed in development of autonomous vehicles for navigating waterways, using a variety of different types of crafts such as automated catamarans [5, 9], small lightweight fanboats [15], kayaks [8] or small inflatable craft [6]. Most of these existing waterway navigation works rely on predefined GPSwaypoints, or a pre-defined map generated from satellite imagery [6]. In contrast our work is focused on autonomous exploration, where the environment is perceived by onboard sensors and the vehicle reacts by planning routes that navigate the vehicle along the waterway and maps the direction and width of the river-bank.

We achieve this with a spinning 3D laser scanner, which has also been demonstrated for local obstacle avoidance [5] and [6] to navigate around obstacles discovered above the water surface. However, we do not use any prior information and rely on intelligent path and goal planning based on the information received by our local sensing. One somewhat related work is by [10] where rivers are detected and tracked from aerial vehicles, although unlike our work these are higher flying vehicles making them as unsuitable as satellite images, whereas our system operates beneath the tree-line, close to the river surface.

In terms of exploration strategies, a common approach is to optimize the robot pose accuracy and the accuracy of the resulting map, [2, 7]. In contrast we rely on separate positioning algorithms [11] for pose accuracy, and instead we focus 
our exploration algorithm to maximize the length of riverbank discovered. In some exploration strategies, information maximization is focused on reducing uncertainty in pose and likelihood of map cells being an obstacle or free space, [2, 7]. Other approaches more closely related to ours define exploration goals that select viewpoints that are expected to yield the highest increase in entropy $[14,13]$ resulting in the robot seeking out regions that have not yet been explored. Overall, these strategies are closely related to the standard frontier exploration systems [16]. Our method is similar in nature, although we introduce a multi-variate cost-map, which finds trajectories that maximize the length of a river explored for a given mission time.

\section{Approach}

The key aim of the work we present here is to plan goals for the vehicle to execute trajectories to realize the following behaviors:

- Follow river, whilst maintaining stable flight and avoiding obstacles

- Maximize the information collected over the course of the river

\subsection{Environment Modeling and Sensing}

The riverine system is modeled as a planar grid $(\chi)$. Each cell $\chi^{i}$ in the grid represents a cell in the world at location $x_{i}, y_{i}$, and the rivermap values of this cell in the grid is as follows:

$$
\chi_{r}^{i}=\chi_{r}^{x_{i}, y_{i}}= \begin{cases}1 & \text { if the cell is part of river } \\ -1 & \text { if the cell is part of bank } \\ 0 & \text { if the cell has not been observed }\end{cases}
$$

Taking $\chi$ we form a function that defines the current information that we have of the river. We define the intersection between river and bank as the pertinent information for mapping the width and course and use these cells as a measure of information. To achieve this we form a new information map as follows to search for discontinuities in the current river model:

$$
I^{\left(x_{i}, y_{i}\right)}=\sum_{u=x_{i}-1}^{u=x_{i}+1} \sum_{v=y_{i}-1}^{v=y_{i}+1}\left(\operatorname{sign}\left(\chi_{r}^{\left(x_{i}, y_{i}\right)}\right) \neq \operatorname{sign}\left(\chi_{r}^{(u, v)}\right)\right)
$$

Our exploration algorithm seeks to extract desirable trajectories for the vehicle that will maximize the entropy in $I$.

The above formulation is derived from data collected from local sensing mounted onboard the vehicle, see Fig.1(c). The laser scanner and the camera onboard gen- 
erate environment maps which are used for goal planning. We segment images received through a camera and use the probabilities generated to create a river map by projecting them onto the river surface using the method from [1] as shown inFig.2.

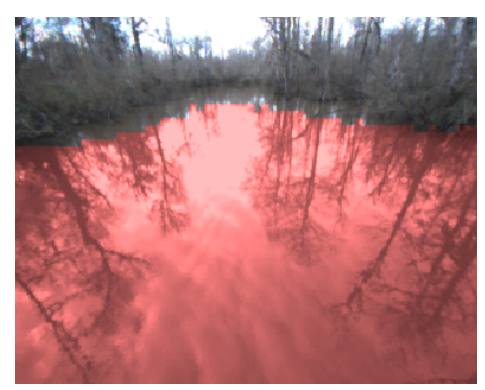

Fig. 2 Pixels with probability greater than .5 after river segmentation are classified as river and marked red [1]. They are then projected onto river plane to create an initial map of the environment. This image is from a dataset taken from McCarthy river, Mississipi.

We use a lightweight spinning laser range scanner to create a 3D scan of the environment (see Fig.1(c) and [12]). The maximum range of this scanner depends on ambient illumination and reflectance characteristics of objects, but in typical outdoor environments we observe maximum ranges of approximately 15 meters. We use this range to determine which laser missed returns are due to limited range and which are due to water absorbtion (i.e. we can detect the river from these laser misses). The laser range measurements are converted into a 3D point cloud in the world frame using an accurate positioning system that operates in GPS denied environments ([12] and [11]). In addition to global positioning we measure the current height above the river surface, which cannot be derived purely from the global frame, since the height will vary according to current water level. To achieve this we extract specular returns from the water surface in a tight cone directly below the vehicle.

Once the global position and relative height above the surface is known, we can then proceed to use the laser measurements to form our environment map. In particular the following rules are applied:

- All missed laser returns (those with the maximum laser range), that pass through the river plane, are considered as river cells at the intersection of the ray and the river plane $\left\{\chi_{r}^{i}=1\right\}$

- All laser hits less than maximum range are projected on the environment grid and based on the density of these projected hits in a cell, the cell is classified as part of the river bank. $\left\{\chi_{r}^{i}=-1\right\}$ 


\subsection{Goal Planning for River Following}

The main task in autonomous exploration is to take local perception of the environment and to extract goals for the vehicle to traverse towards. The goals are then fed in as input to the low-level motion planning algorithm. The low-level motion planning we have developed in earlier in [4]. The exploration algorithm we present here sets goals that seek to maximize the information gained during the mission.

To achieve desired behaviors we introduce multivariate cost maps, that respect the characteristics of the sensing and extend the abilities of more simplistic traditional frontier exploration algorithms [16]. In particular the costs we derive enable the vehicle to observe the maximum amount of the riverbank whilst following the course of the river, and where possible avoid returning to unexplored portions of the river that are behind the vehicle. Unexplored frontier that was not observed as the vehicle passed by initially, may become larger in size than a narrow passage that the vehicle encounters directly ahead, however, it is suboptimal to return to these locations as little new information is collected on the journey back to previously explored areas.

For one we develop a riverbank hugging behavior which uses a distance transform based cost function, $C_{d}(\cdot)$ that aims to keep the vehicle away-from but nearenough distance to the riverbank to both assist the 3D mapping of the bank and ensure the local motion estimation is functional. This range is designed to result in maximal information gain of the riverbank. To arrive at $C_{D}(i)$ we compute a distance transform $f_{D}(i)$ that returns the distance to the nearest obstacle $\left(\chi_{r}^{j}<0\right)$. We very efficiently compute this distance cost as described in detail in [12]. After calculating the distance transform we apply a function to penalize goals very close to obstacles and also penalize goals far away from obstacles using a desired distance $\kappa_{D}$ as follows:

$$
\begin{aligned}
& C_{D}(i)=1-\exp \left(k_{D}\left(f_{D}(i)-\kappa_{D}\right)^{2}+\frac{1}{f_{D}(i)}\right) \\
& f_{D}(i)=\underset{\substack{j=1: N \\
\chi_{r}^{j}<0}}{\operatorname{argmin}}\left\|\left(x_{i}, y_{i}\right)-\left(x_{j}, y_{j}\right)\right\|
\end{aligned}
$$

where $k_{d}$ is a tuning constant. The resulting functional is depicted in Fig. 3(a), where the cost is high near the obstacles and descends to a minima at $\kappa_{D}$.

The next cost we introduce is designed to avoid retracing steps, in particular we assign cells that have been observed more recently with lower cost than those behind the vehicle, that were observed further in the past. We take the elapsed time since the $i$ th cell was last observed as $\chi_{t}^{i}$ and use it to penalize retracing through cells seen previously as follows:

$$
C_{T}(i)=t-\chi_{t}^{i}
$$

Fig. 3(b), visualizes this temporal observation cost. An important cost we introduce is $C_{R}(i)$. The range the cell is from the current vehicle-location, which is designed to maximize distance traversed along river: 


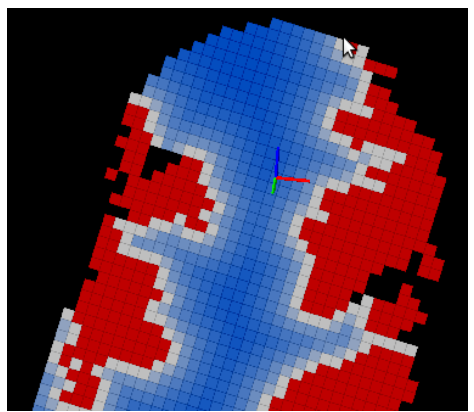

(a) Distance transform

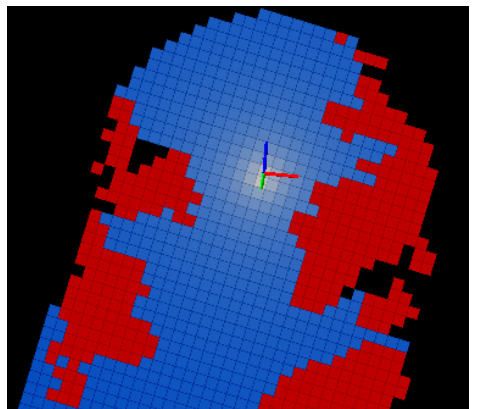

(c) Range of the cell to the current vehicle location

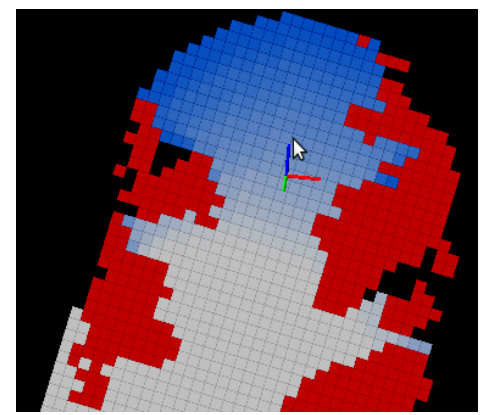

(b) Relative temporal differences between observations

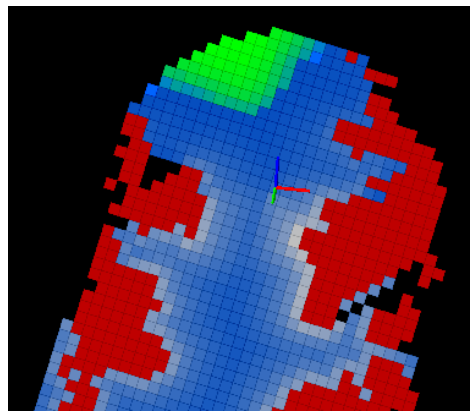

(d) Combined multi-variate cost function

Fig. 3 Visualizations of the multi-variate cost functions. Obstacles are highlighted in red, the cells observed as river are rendered a shade from white to blue, where deep blue represents low cost to go. Cells with lowest cost in a map represent the next goal point for navigation. For the combined cost functions we indicate the cluster of lowest cost cells in green.

$$
C_{R}(i)=\left\|\left(x_{i}, y_{i}\right)-\left(x_{t}, y_{t}\right)\right\|
$$

where $\left(x_{t}, y_{t}\right)$ is the current position of the vehicle, see Fig. 3(c).

Next we introduce a cost to favor the vehicle continuing on its current course to avoid the issue of isotropic sensor input that typically occurs at commencement of a mission when no obstacles are within range and the aforementioned costs are at an equilibrium and do not return stable goals.

$$
\begin{aligned}
C_{H}(i) & =\exp ^{\kappa_{H}\left(\theta_{z}^{v}-\Delta_{\theta}\right)} \\
\Delta_{\theta} & =\arctan \left(\left(\frac{x_{t}-x_{i}}{y_{t}-y_{i}}\right)^{2}\right)
\end{aligned}
$$

Where $k_{H}$ and $\kappa_{H}$ are constants that are empirically determined to create a dip in cost around zero heading to enable vehicle to maintain its course when the sensory inputs do not provide stable goals, such as in open waters. 
Finally an obstacle path cost $C_{O}(i)$ is derived from the set of cells $(P)$ connecting the vehicle position with cell $i$ :

$$
\begin{aligned}
& C_{O}(i)=\underset{p \in P}{\operatorname{argmax}}\left(f_{O}(p)\right) \\
& f_{O}(p)= \begin{cases}0 & \text { if } \chi_{r}^{p}==1 \\
\kappa_{O} & \text { otherwise }\end{cases}
\end{aligned}
$$

Where $\kappa_{O}$ is a suitably large constant to avoid obstacles. Individually these costs do not produce desirable behavior, however when correctly fused together, the vehicle maintains course. Therefore, the final objective of the goal planning algorithm is to combine the costs and extract the resulting goal $\chi^{G}$ that is to be passed to the motion planning algorithm.

Then to extract goals we compile a set $\Psi$ that contains the cells with the lowest $n \%$ cost, then find a weighted mean over this set:

$$
G=\underset{\psi \in \Psi}{\operatorname{argmin}}\left(\sum_{i=1: N}\left(\left\|\mid\left(x_{\psi}, y_{\psi}\right)-\left(x_{i}, y_{i}\right)\right\| \cdot C(\psi)\right)\right)
$$

\section{Results}

We validate our method in a set of experiments, beginning with controlled simulations executed within maps of real-world data, continuing with results from autonomous flights over rivers and waterways and then we present open loop comparison with human operator on real-world data.

\subsection{Simulated Exploration on Real-world Data}

For simulation we use an environment model generated from data collected over a section of McCarthy River in Mississippi, USA. A 3D point cloud registered in world coordinates is generated from data collected through the sensor suite carried on a boat traversing the river. From this point cloud we can simulate the laser measurements given a particular pose of the robot and the known characteristics of our laser scanner. This gives us the means to evaluate our autonomous exploration algorithm based on multi-variate cost maps against a traditional frontier exploration algorithm.

We use planning cycles executed every 10 seconds and use a fixed number of planning cycles to give a fair evaluation. Each algorithm is given the same initial conditions and we measure the information gained at each time step during the simulated mission. In Fig. 4 the information gained with approach is plotted against time, where clearly the more traditional frontier approach has difficulties maintain- 
ing advantageous trajectories for mapping the riverbank. When narrow passages appear in the river, the frontier algorithm oscillates between returning to explore the earlier unexplored frontier segments and returning to the narrow passages. Our method both maintains optimal distance from the riverbank to avoid suboptimal trajectories and also selects trajectories with higher probability of maintaining course along the river and avoid backtracking down the river to observe portions of the bank.

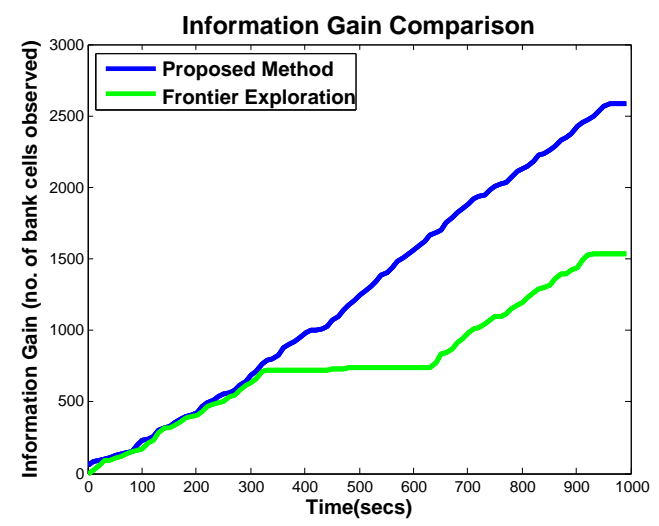

Fig. 4 Simulation of autonomous exploration comparing our method using multi-variate cost maps against a more traditional frontier exploration algorithm [16]. The simulation is given the same initial conditions within an environment model formed from real-data collected from a section of McCarthy River in Mississippi, USA. The information gained using each algorithm is compared. When presented with a narrow passage appears, the frontier algorithm oscillates between returning to earlier unexplored segments of the river, whereas our algorithm continues in the trajectory that is far greater probability of increasing the information collected of the riverbank.

\subsection{Autonomous Flights}

After demonstrating in simulation that our exploration algorithm is more optimal at returning favorable trajectories, we now proceed to evaluate our approach in realworld truly autonomous flights over rivers and waterways. We manually bring our system into a hover over a river and switch into autonomous mode and from there let the vehicle explore the river autonomously at a velocity of $1 \mathrm{~m} / \mathrm{s}$ with no further human inputs.

The flights are on tight and densely vegetated sections of a river, and demonstrate the complete system for planning trajectories that avoid overhanging tree obstacles and maintaining course along the river. The algorithm is able to plan trajectories which enable the system to stay in range of both riverbanks where possible resulting in an optimal trajectory for maximizing information. 


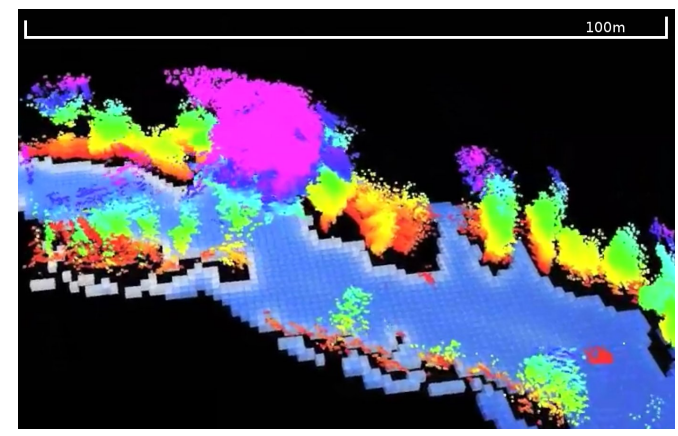

(a)

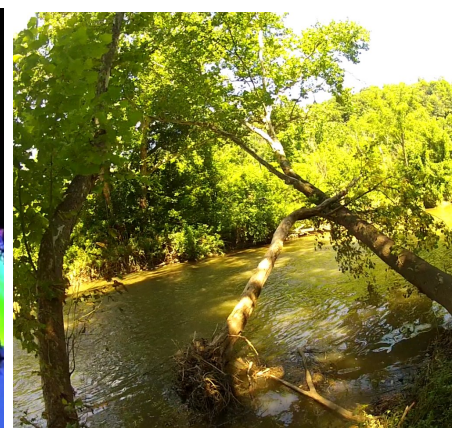

(b)

Fig. 5 (a)Laser-point cloud collected from the autonomous flight through the densely-forested river environment. The detected river extent is colored with blue cells and the bank and overhanging trees are colored by height above river surface. (b) Environment where we test the system's ability to fly autonomously through a densely forested river.

Fig. 5 shows an example of the cluttered river environment the robot is flying through during an autonomous $100 \mathrm{~m}$ flight. The canopy and the river/bank classification map from this flight are shown in Fig. 6. This experiment demonstrates the advantage of our algorithm against getting information from satellite images as we are able to classify the areas lying underneath the canopy as river or bank.

Finally we demonstrate the ability of the system to navigate a river over long distances. In the longest autonomous run on a river, the robot flew for about $450 \mathrm{~m}$ along the length of a narrow river. The limiting factor in the distance covered during this test was the battery life of the vehicle.

This experiment was conducted on a very shallow river about $10-15 \mathrm{~m}$ in width with dense vegetation on both banks. The robot localizes itself without any GPS input and is able to classify the cluttered environment into river and obstacles to explore and plan through it. There were some pauses in robot trajectory in some sections of the river due to trees with branches hanging over the river blocking the path for the robot. Shallow areas in the river made the problem harder as they would be classified as obstacles due to large number of laser returns. Wind was also a challenge as a slight drift from the trajectory would take the robot too close to an obstacle. The final map of the bank and the trajectory of the vehicle during this experiment is displayed in Fig. 7. The system operates without manual intervention successfully exploring the river and turning according to the river direction.

\subsection{Open Loop Comparison}

Finally, we compare vision and laser sensors for navigation against a human operator, whom we consider to make expert decisions on how to navigate along the river. Human decisions are either left or right turn decisions which are measured 


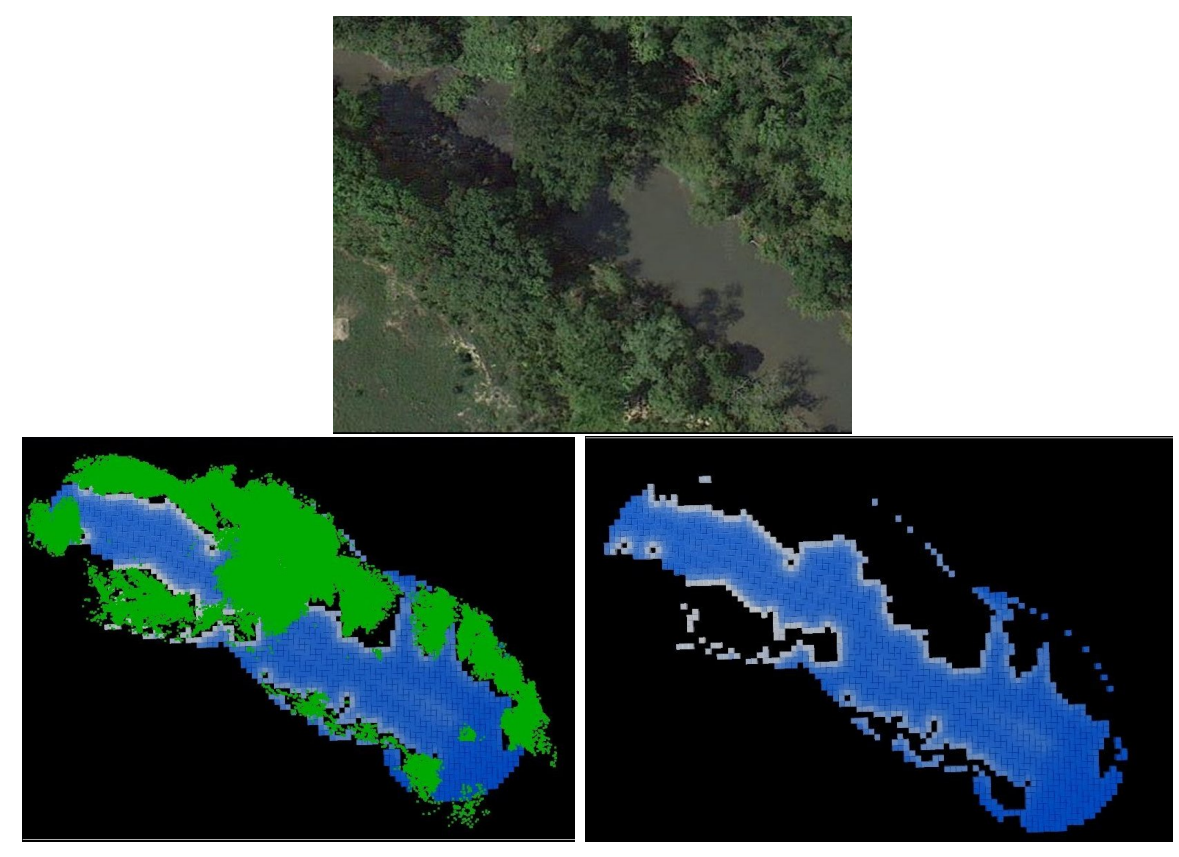

Fig. 6 Map generated from autonomous flight through narrow and densely vegetated riversegment. Top: Satellite view of river segment. Left: Map of river where green is the bank and overhanging trees detected by the laser scanner. Right: Traversable river extent detected by the laser scanner. Notice the overhanging trees in the middle of the segment are removed and a clear and traversable path is discovered underneath by the flying vehicle. The traversable river map, for example, could be used by a surface craft following the flying vehicle enabling it to have knowledge of where it is safe to travel.

from heading changes in pose estimate. We perform an open loop comparison of the sensors and human operator on data collected by the robot platform fixed on a boat driven along McCarthy river.

We compare the decisions made by the human operator against the turn decisions made based on goal-points received from the multi-variate exploration algorithm using laser as well as vision sensing.

Since the laser scanner has a short range, laser based navigation follows the contours of a bank closely making more reactive decisions. The vehicle is able to look further ahead using vision to make intelligent and time-efficient decisions. This difference is emphasized in a wide river, where vision will lead the vehicle down the river following its general course, but laser navigation will stick to the bank and follow its contours which is time-inefficient and in contradiction to what a human operator would do. After an initial $50 \mathrm{~m}$, the next $150 \mathrm{~m}$ stretch is more than $25 \mathrm{~m}$ wide and vision performs much better than laser for this stretch, see Fig. 8(b), (c). 


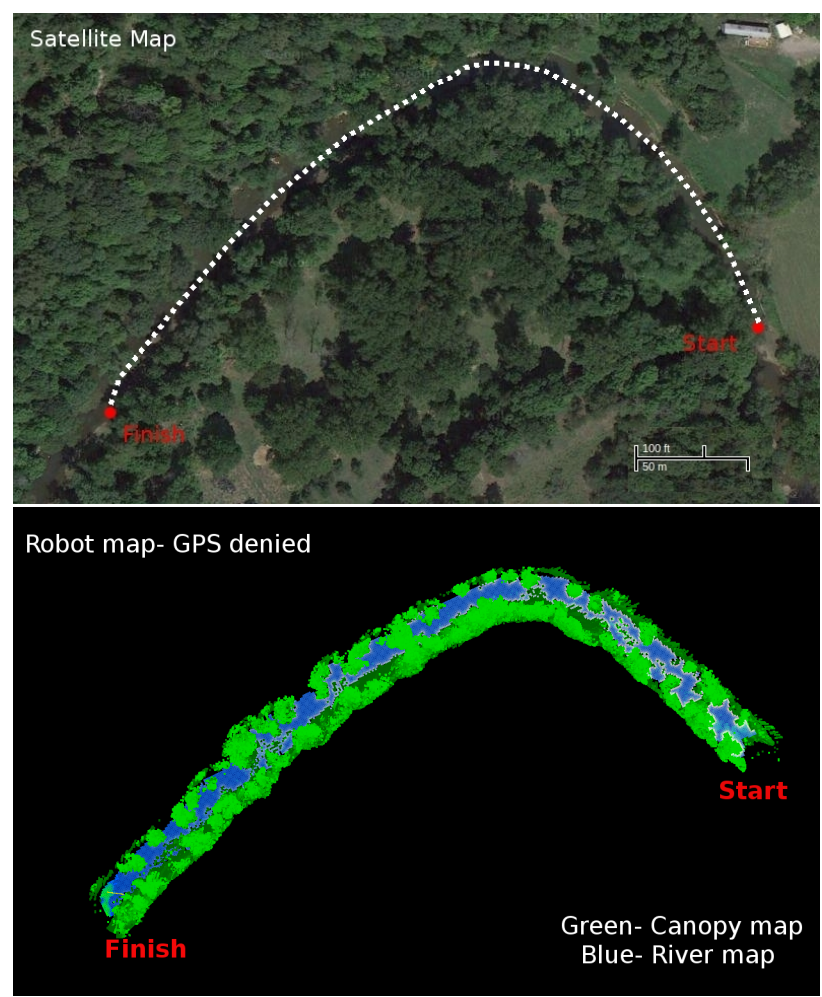

Fig. 7 Autonomous flight: Top: Satellite image with overlaid flight trajectory and Bottom: The river/bank map (blue) and canopy map (green) generated by the robot from data collected in a $450 \mathrm{~m}$ autonomous flight along a river.

\section{Conclusion}

Our work demonstrates that autonomous autonomous exploration is possible in river environments and that neither GPS waypoints nor prior maps are necessary. In addition we also demonstrate GPS denied flight with planning algorithms that can robustly extract goal points in challenging unstructured terrain. While there is much work in autonomous exploration for ground-vehicles, these algorithms do not directly translate to river environments. Our system is developed to respect the specific physical layout and properties of the river and bank and the behavior of the sensors and perception algorithms in these environments.

In future work, we still see challenges to increase the operating velocity in a safe manner. One avenue to explore is to predict ahead the course of the river with more accuracy giving confidence of which directions are most likely to possess freespace and when turns, dead-ends or forks are likely to appear. We also see persistent monitoring a waterway as an important means to detect pertinent changes to the environment. Further we see that small flying vehicles while fast and nimble, have 

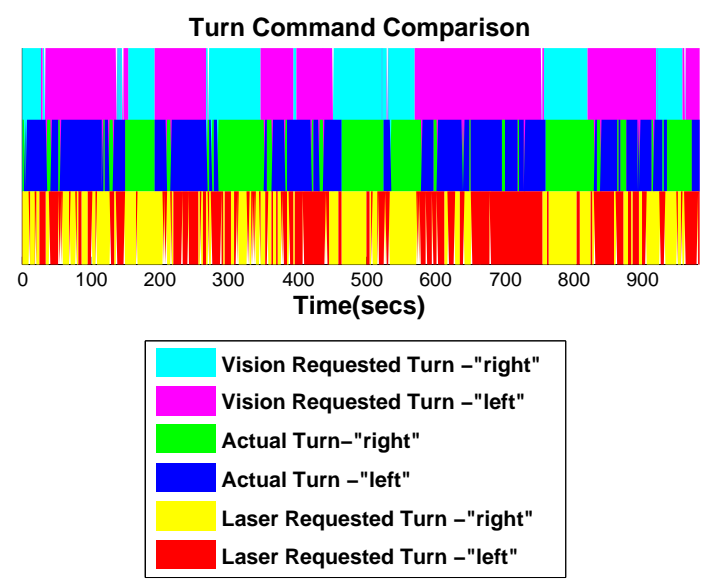

(a)

\begin{tabular}{|l|c|r|}
\hline & $50-200 \mathrm{~m}$ & full path \\
\hline Vision & $79.3 \%$ & $77 \%$ \\
\hline Laser & $54 \%$ & $66 \%$ \\
\hline
\end{tabular}

(b)

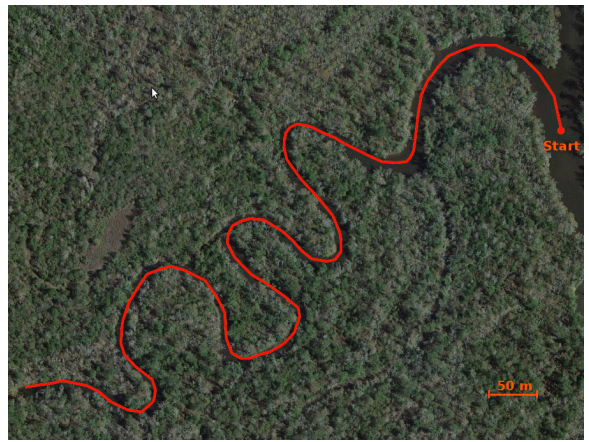

(c)

Fig. 8 (a) Comparison of turn commands over time. Long range vision commands are more stable and do not require the vehicle to change directions unnecessarily. Laser commands are more jittery as they consider a much smaller environment while planning and react suddenly to any changes. (b) \% accuracy of vision and laser turn requests by comparing them to the ground truth. Results from the 50-200m wide stretch show a larger difference in vision and laser accuracies. (c)The 1.5 $\mathrm{km}$ path followed on McCarthy river.

limited time of flight and must be combined with a supporting vehicle which is larger and trails behind offering the ability for the flying vehicle to return for landing and recharging. These non-homogenous teams of vehicles pose many interesting research challenges, in both high fidelity localization and tracking and with relative motion planning for high-speed take-offs and landings ,in addition to information sharing to exploit the different sensing characteristics and viewing perspectives of the vehicles. 


\section{References}

1. Achar, S., Sankaran, B., Nuske, S., Scherer, S., Singh, S.: Self-supervised segmentation of river scenes. In: 2011 IEEE International Conference on Robotics and Automation, May, pp. 6227-6232. Ieee (2011). DOI 10.1109/ICRA.2011.5980157

2. Amigoni, F., Caglioti, V.: An information-based exploration strategy for environment mapping with mobile robots. Robotics and Autonomous Systems 58(5), 684-699 (2010). DOI 10.1016/j.robot.2009.11.005

3. Chambers, A., Achar, S., Nuske, S., Rehder, J., Kitt, B., Chamberlain, L., Haines, J., Scherer, S., Singh, S.: Perception for a river mapping robot. In: 2011 IEEE/RSJ International Conference on Intelligent Robots and Systems, pp. 227-234. Ieee (2011). DOI 10.1109/IROS.2011.6095040

4. Cover, H., Choudhury, S., Scherer, S., Singh, S.: Sparse tangential network (spartan): Motion planning for micro aerial vehicles. In: International Conference on Robotics and Automation (2013)

5. Dunbabin, M., Grinham, A., Udy, J.: An autonomous surface vehicle for water quality monitoring. In: Australasian Conference on Robotics and Automation (ACRA) (2009)

6. Gadre, A., Du, S., Stilwell, D.: A topological map based approach to long range operation of an unmanned surface vehicle. In: American Control Conference, June, pp. 5401-5407 (2012)

7. Kollar, T., Roy, N.: Trajectory optimization using reinforcement learning for map exploration. The International Journal of Robotics Research (June) (2008)

8. Leedekerken, J., Fallon, M., Leonard, J.: Mapping complex marine environments with autonomous surface craft. In: The 12th International Symposium on Experimental Robotics (2010)

9. Pradalier, C., Posch, T., Pernthaler, J., Siegwart, R.: Design and Application of a Surface Vessel for Autonomous Inland Water Monitoring. IEEE Robotics \& Automation Magazine pp. 1-9 (2012)

10. Rathinam, S., Almeida, P., Kim, Z., Jackson, S., Tinka, A., Grossman, W., Sengupta, R.: Autonomous Searching and Tracking of a River using an UAV. In: American Control Conference, pp. 359-364. Ieee (2007). DOI 10.1109/ACC.2007.4282475

11. Rehder, J., Gupta, K., Nuske, S., Singh, S.: Global pose estimation with limited gps and long range visual odometry. In: Proceedings of the 2012 IEEE/RSJ International Conference on Robotics and Automation (2012)

12. Scherer, S., Rehder, J., Achar, S., Cover, H., Chambers, A., Nuske, S., Singh, S.: River mapping from a flying robot: state estimation, river detection, and obstacle mapping. Autonomous Robots 33(1-2), 189-214 (2012). DOI 10.1007/s10514-012-9293-0

13. Stachniss, C., Burgard, W.: Exploring unknown environments with mobile robots using coverage maps. In: International Joint Conference on Artificial Intelligence, pp. 1127-1132 (2003)

14. Stewart J. Moorehead and Reid Simmons and William L. Whittaker: Autonomous exploration using multiple sources of information. In: IEEE International Conference on Robotics and Automation (2001)

15. Valada, A., Velagapudi, P., Kannan, B., Tomaszewski, C., Kantor, G.A., Scerri, P.: Development of a low cost multi-robot autonomous marine surface platform. In: The 8th International Conference on Field and Service Robotics (FSR 2012) (2012)

16. Yamauchi, B.: A frontier-based approach for autonomous exploration. In: Computational Intelligence in Robotics and Automation, 1997. CIRA'97., Proceedings., 1997 IEEE International Symposium on, pp. 146-151. IEEE Comput. Soc. Press (1997). DOI 10.1109/CIRA.1997.613851 